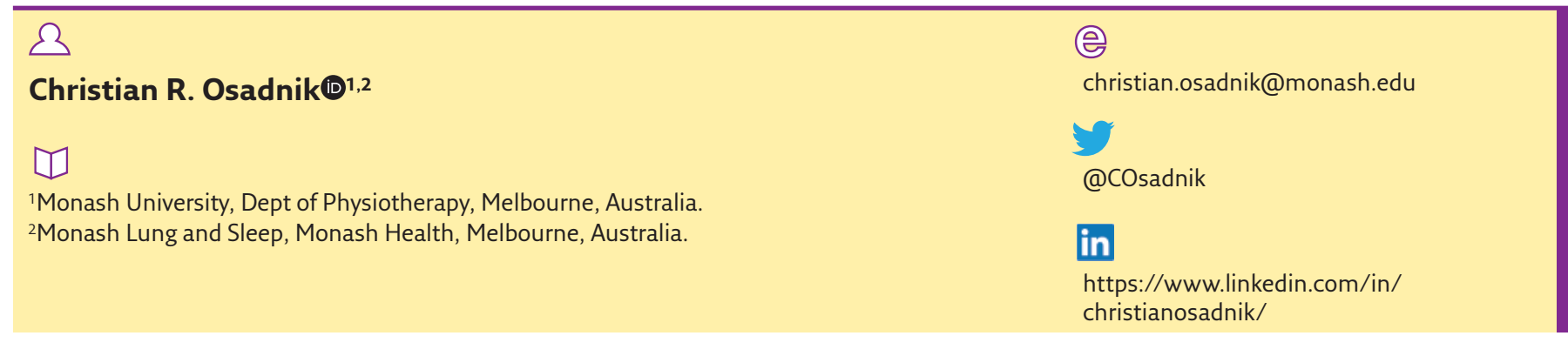

\title{
Cardiorespiratory physiotherapy training and practice in Australia
}

\section{Physiotherapy in Australia}

In 2018, Australia's population of approximately 25 million people is serviced by almost 32000 registered physiotherapists [1]. Physiotherapists in Australia traditionally obtain their qualification via a 4-year, full-time equivalent undergraduate professional degree. This typically involves 2 or 3 years of full-time, university-based education followed by full-time clinical education, during which the student is immersed in real-world clinical training under the supervision of physiotherapy clinicians.

Like their colleagues in many other parts of the world, Australian physiotherapists have a broad scope of practice. We work within an integrated healthcare system alongside nursing, allied health and medical teams, but are considered primary contact practitioners. This means patients do not need medical referrals to access physiotherapy services, and we have autonomy to make independent decisions regarding patient assessments and treatments within our scope of practice. We are also not restricted with respect to our particular area of practice. Newly graduated physiotherapists can work in the public or private sector, in hospitals or clinics, with paediatric or geriatric patients, and in areas ranging from respiratory medicine to intensive care to neurology to musculoskeletal physiotherapy to women's health.

In order to legally practice in Australia, physiotherapists must be registered with the Physiotherapy Board of Australia, part of the Australian Health Practitioner Regulation Agency (https://www.ahpra.gov.au/), and abide by their codes of conduct. Physiotherapy education and training standards are closely regulated by the Australian Physiotherapy Council (https://physiocouncil.com.au/), which is also the body responsible for conducting examinations of overseas-trained physiotherapists who wish to practice in Australia. The physiotherapy profession is supported by our peak national body, the Australian Physiotherapy Association (APA) (www. physiotherapy.asn.au).

\section{Clinical respiratory physiotherapy practice}

Most physiotherapists who practice in respiratory medicine work within the public healthcare system, as this is where most patient care is delivered. Many work in acute and/or rehabilitation hospitals, with a large proportion also practising in outpatient or community services. Some of our main areas of practice are:

- acute respiratory care for patients admitted with acute or acute-on-chronic respiratory disease (e.g. chronic obstructive pulmonary disease, asthma and bronchiectasis)

- post-surgical care (e.g. thoracic, cardiac and abdominal surgery)

- higher dependency/specialist care (e.g. intensive care and tracheostomy care)

- general medicine

- outpatient pulmonary rehabilitation and heart failure

- cystic fibrosis

Private hospitals may offer similar patient services to that of public hospitals; however, private practices providing respiratory physiotherapy services are not common. "Extended scope of practice" physiotherapy services in respiratory medicine are emerging. A small group of clinicians with specific expertise are testing the professional
Cite as: Osadnik CR. Cardiorespiratory physiotherapy training and practice in Australia. Breathe 2018; 14: 243-245. 
waters with forays into areas of a more invasive nature (e.g. advanced tracheostomy care, intubation and bronchoscopy).

\section{Respiratory-specific physiotherapy training in Australia}

Cardiorespiratory medicine is one of the core areas in which all physiotherapists must demonstrate competency in order to receive a physiotherapy qualification in Australia. Respiratory medicine therefore falls under the umbrella of "cardiorespiratory", the more commonly used term here. All physiotherapy courses require students to study cardiorespiratory theory at university and undertake clinical education that provides experience in cardiorespiratory medicine. A cardiorespiratory physiotherapy clinical placement may vary in length between differing academic institutes, but would typically last 4-6 weeks. During this time, students are likely to receive direct experience managing patients with respiratory-specific diseases; however, some clinical settings lend themselves to a greater focus on other areas along the continuum of practice. For example, cardiorespiratory clinical experience may be obtained in the areas of medical, surgical or outpatient care based in acute, rehabilitation or community settings. There has been recent interest in substitution of components of clinical education with clinical simulation (e.g. computerised mannequins in simulation laboratories or actor/peer-led role play clinical scenarios) in order to enhance students' pre-clinical skills and reduce the education burden imposed upon healthcare providers.

\section{Physiotherapy specialisation in Australia}

Physiotherapy in Australia is tightly regulated with respect to the terminology used to refer to our own profession. The large majority of physiotherapists are just "physiotherapists". Some members of the APA are eligible to apply for "titling" (achieved through experiential or academic pathways), which allows them to use the term "APA Cardiorespiratory Physiotherapist". A smaller group of highly experienced clinicians choose to apply for formal specialisation through the Australian College of Physiotherapists (under the auspices of the APA), which allows them to use the title "Specialist Cardiorespiratory Physiotherapist". These are the only two exceptions allowing one to refer to themselves as anything but a "physiotherapist" in Australia. Completion of a postgraduate research degree such as a Doctor of Philosophy provides a significant boost to one's academic and clinical standing within the profession, and allows for the use of "Dr" before one's name and the addition of postnominal letters. Such individuals must, however, only refer to themselves as a "physiotherapist". Climbing the professional ladder towards positions of seniority and leadership can, of course, occur without specialisation.

\section{The emergence of different training models (and its associated challenges)}

The Australian physiotherapy education climate has evolved significantly over the past decade into a highly competitive national and international marketplace. This has resulted in considerable modification to traditional course structures. We now have more than 20 accredited courses operating throughout Australia in 2018, with many operating "hybrid" models that necessitate crediting for prior recognition of learning in core areas. For example, some courses are now only able to be commenced by students who are entering into studies as a postgraduate student, after completion of an approved related degree. The diversity of physiotherapy education programmes has led to an equally diverse array of qualifications. The humble "Bachelor of Physiotherapy" qualification is now one of many that enable entry-level physiotherapy registration. Some universities offer Bachelor degrees; others provide a Masters qualification. Some Bachelor degrees may be completed with or without an "honours" component, which may or may not denote completion of a research thesis. Some new graduates can refer to themselves as "Doctor of Physiotherapy" (completion of a Masters (extended) qualification) while others cannot. This heterogeneity unfortunately creates confusion within the healthcare system and the general public to discern what, if any, impact the nuances of these qualification differences have upon the expected standard of patient care. At the end of the day, every new-graduate Australian physiotherapist is just that: a newly graduated physiotherapist who has met the standard to be registered to practice. 


\section{Walking in my shoes}

I am a Senior Lecturer and Research Fellow within the Department of Physiotherapy at Monash University (Melbourne, Australia). I am currently only based on campus one day per week whilst undertaking a postdoctoral fellowship based in the Monash Lung and Sleep department of Victoria's largest healthcare provider, Monash Health. This is quite a rare work situation for a physiotherapist in Australia, as combined clinical/research positions in "university/ hospital" contexts are not commonplace. A large factor underpinning my ability to acquire funding to create such a role was my prior completion of a long-term fellowship through the European Respiratory Society (in 20142015). For this, I was based in the laboratory of Thierry Troosters in Leuven, Belgium, where

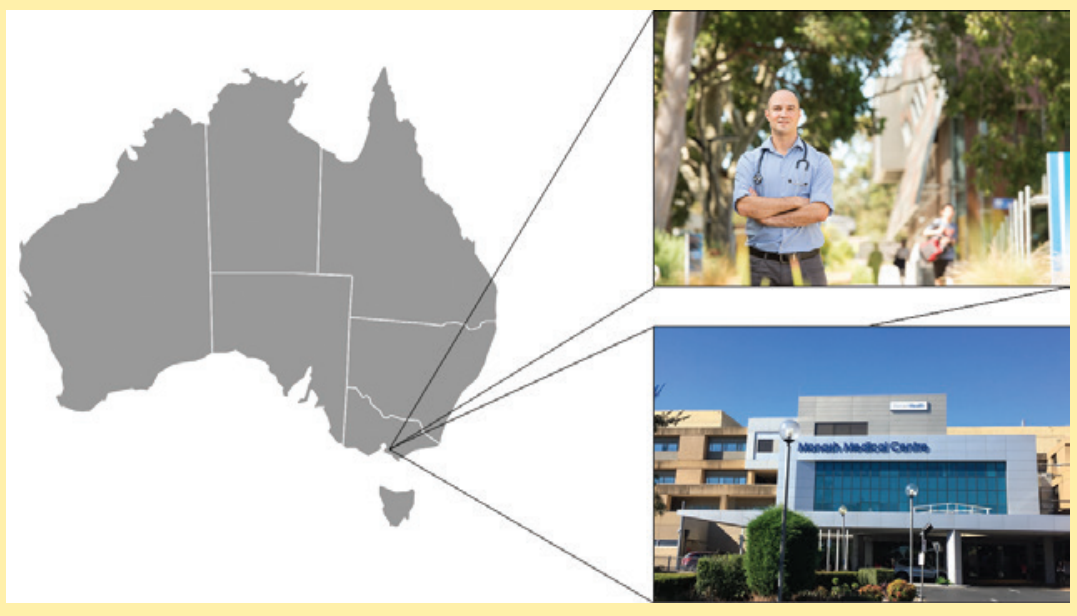

Top: Monash University, Melbourne; bottom: Monash Health, Melbourne.

I conducted research in the pulmonary rehabilitation setting. I place a very high value on my current work arrangement, and hope to remain a vital conduit between clinicians working with patients who are acutely unwell due to respiratory illness and the generation of new research that will hopefully impact upon tomorrow's evidence-based respiratory medicine practice.

In order to achieve this, my typical day commences by spending mornings on the acute respiratory wards. This is mostly for the screening of patients with chronic lung disease for recruitment into my studies. I work closely with the whole medical, nursing and allied health respiratory team, and have a great network of collaborators, research assistants and students within my team. I collect patient data via questionnaires, physiotherapy testing or database searching for my current projects, but do not manage a clinical caseload. My afternoons commonly involve data analysis and/or manuscript writing and attending meetings. We have a weekly lunchtime "grand round" meeting, which is a great place to keep up with current departmental initiatives and the latest research within respiratory medicine. I also spend time with the physiotherapy clinical staff in pulmonary rehabilitation twice per week for project data collection. I base myself at Monash University in the Department of Physiotherapy one day per week where I deliver teaching activities to undergraduate physiotherapy students, facilitate student research projects and mentor junior academic colleagues. In between these roles, I manage a busy, but rewarding, portfolio that includes committee responsibilities for national and international respiratory societies, editorial responsibilities for Cochrane Airways, coordination of cardiorespiratory physiotherapy professional development activities and academic administration tasks.

\section{Acknowledgements}

I wish to thank Prue Morgan, head of Monash University Department of Physiotherapy, for her assistance to accurately describe physiotherapy regulations in Australia and for critical revision of the final manuscript.

\section{Conflict of interest}

None declared.

\section{References}

1. Physiotherapy Board of Australia. Physiotherapy Board of Australia Registrant Data, reporting period 1 January 2018 31 March 2018. www. physiotherapyboard.gov.au/About/
Statistics.aspx. Date last updated: April 18, 2018. Date last accessed: May 1, 2018. 